\title{
Population Pharmacokinetics and Dosing Optimization of Amoxicillin in Neonates and Young Infants
}

\author{
Bo-Hao Tang, ${ }^{\text {a } Y u e-E ~ W u, ~}{ }^{\text {a Chen Kou, }}$

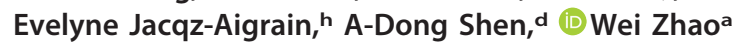

aDepartment of Clinical Pharmacy, School of Pharmaceutical Sciences, Shandong University, Jinan, China

bDepartment of Neonatology, Beijing Obstetrics and Gynecology Hospital, Capital Medical University, Beijing, China

cNeonatal Intensive Care Unit, Beijing Children's Hospital, Capital Medical University, National Center for Children's Health, Beijing, China

dBeijing Key Laboratory of Pediatric Respiratory Infection Diseases, Key Laboratory of Major Diseases in Children, Ministry of Education, National Clinical Research Center for Respiratory Diseases, National Key Discipline of Pediatrics (Capital Medical University), Beijing Pediatric Research Institute, Beijing Children's Hospital, Capital Medical University, National Center for Children's Health, Beijing, China

eDepartment of Neonatology, Shandong Provincial Qianfoshan Hospital, Shandong University, Jinan, China

fNeonatal Intensive Care Unit, CHU de Rennes, Rennes, France

gDepartment of Pharmacy, Shandong Provincial Qianfoshan Hospital, Shandong University, Jinan, China

hDepartment of Pediatric Pharmacology and Pharmacogenetics, Hôpital Robert Debré, APHP, Paris, France

ABSTRACT Amoxicillin is widely used to treat bacterial infections in neonates. However, considerable intercenter variability in dosage regimens of antibiotics exists in clinical practice. The pharmacokinetics of amoxicillin has been described in only a few preterm neonates. Thus, we aimed to evaluate the population pharmacokinetics of amoxicillin through a large sample size covering the entire age range of neonates and young infants and to establish evidence-based dosage regimens based on developmental pharmacokinetics-pharmacodynamics. This is a prospective, multicenter, pharmacokinetic study using an opportunistic sampling design. Amoxicillin plasma concentrations were determined using high-performance liquid chromatography. Population pharmacokinetic analysis was performed using NONMEM. A total of 224 pharmacokinetic samples from 187 newborns (postmenstrual age range, 28.4 to 46.3 weeks) were available for analysis. A two-compartment model with first-order elimination was used to describe population pharmacokinetics. Covariate analysis showed that current weight, postnatal age, and gestational age were significant covariates. The final model was further validated for predictive performance in an independent cohort of patients. Monte Carlo simulation demonstrated that for early-onset sepsis, the currently used dosage regimen $(25 \mathrm{mg} / \mathrm{kg}$ twice daily [BID]) resulted in $99.0 \%$ of premature neonates and $87.3 \%$ of term neonates achieving the pharmacodynamic target (percent time above MIC), using a MIC breakpoint of $1 \mathrm{mg} /$ liter. For late-onset sepsis, $86.1 \%$ of premature neonates treated with $25 \mathrm{mg} / \mathrm{kg}$ three times a day (TID) and $79.0 \%$ of term neonates receiving $25 \mathrm{mg} / \mathrm{kg}$ four times a day (QID) reached the pharmacodynamic target, using a MIC breakpoint of $2 \mathrm{mg} /$ liter. The population pharmacokinetics of amoxicillin was assessed in neonates and young infants. A dosage regimen was established based on developmental pharmacokinetics-pharmacodynamics.

KEYWORDS amoxicillin, dosing optimization, neonates, pharmacokinetics

moxicillin, a penicillinase-susceptible semisynthetic amino-penicillin, is a broadspectrum antibiotic that is active against both Gram-negative and Gram-positive pathogens and inhibits $\beta$-lactamase-free strains of Listeria monocytogenes, Haemophilus
Citation Tang B-H, Wu Y-E, Kou C, Qi Y-J, Qi H, Xu H-Y, Leroux S, Huang $X$, Zhou Y, Zheng $Y$, Jacqz-Aigrain E, Shen A-D, Zhao W. 2019. Population pharmacokinetics and dosing optimization of amoxicillin in neonates and young infants. Antimicrob Agents Chemother 63:e02336-18. https://doi.org/10.1128/AAC .02336-18.

Copyright $\odot 2019$ American Society for Microbiology. All Rights Reserved.

Address correspondence to Wei Zhao, zhao4wei2@hotmail.com.

B-H.T., Y-E.W., and C.K. contributed equally to this article.

Received 6 November 2018

Returned for modification 19 November 2018

Accepted 27 November 2018

Accepted manuscript posted online 3 December 2018

Published 29 January 2019 
TABLE 1 Baseline characteristics in 187 neonates and infants ${ }^{a}$

\begin{tabular}{lll}
\hline Parameter $^{b}$ & Mean (SD) & Median (range) \\
\hline GA (wks) & $37.6(2.51)$ & $38.1(28.3-41.4)$ \\
PMA (wks) & $38.4(2.61)$ & $39.0(28.4-46.3)$ \\
PNA (days) & $7.47(6.06)$ & $7.00(1.00-37.0)$ \\
Birth wt (kg) & $2.93(0.716)$ & $3.05(1.04-4.60)$ \\
Current wt (kg) & $2.99(0.733)$ & $3.21(1.06-4.58)$ \\
Serum creatinine concn ( $\mu$ mol/liter) & $48.7(28.7)$ & $38.0(8.80-156)$ \\
Amoxicillin treatment regimen & & \\
$\quad$ Duration (days) & & \\
$\quad$ Dose (mg) & $6.00(3.95)$ & $7.00(1.00-37.0)$ \\
$\quad$ Unit dose (mg/kg/dose) & $70.5(17.9)$ & $23.0(15.0-100)$ \\
\hline
\end{tabular}

${ }^{a}$ The patient population consisted of 98 male patients and 89 female patients. All patients were Chinese.

${ }^{b} \mathrm{GA}$, gestational age at birth; PMA, postmenstrual age; PNA, postnatal age.

influenzae, Salmonella species, and Escherichia coli $(1,2)$. It has a low serum protein binding level and is excreted primarily through glomerular filtration (1). It follows a time-dependent bacterial killing mechanism, for which the pharmacokineticspharmacodynamics (PK-PD) relationship is the time period $(T)$ in which the free antimicrobial drug concentration is above the MIC $\left(f T_{>M I C}\right)$. Despite amoxicillin being generally considered safe due to its wide therapeutic range, excessive accumulation of amoxicillin can lead to seizures and crystalluria (3).

Amoxicillin is among the most commonly used antibiotics for the treatment of bacterial infections in neonates. However, considerable intercenter variability in dosage regimens exists in neonatal clinical practice (4). To the best of our knowledge, the pharmacokinetics of amoxicillin has been described in only a few preterm infants $(2$, 5-7), and no pharmacokinetics-pharmacodynamics-based optimum dosing recommendations have been made. A model-based dosing recommendation was available only for term neonates undergoing hypothermia (8). It has been clearly shown that hypothermia has a profound impact on the pharmacokinetics of antimicrobial agents and that hypothermic patients should follow special dosing recommendations, which cannot be extrapolated to the general population (8). The lack of pharmacokinetic data of the target population may increase the risk of the irrational use of antimicrobial agents, which could further encourage the overgrowth of pathogens and the spread of clinical antibiotic resistance.

Therefore, we aimed to conduct a prospective, multicenter pharmacokinetic study using a large sample size that included the entire age range of neonates and young infants in order to assess the population pharmacokinetics of amoxicillin and also to develop an evidence-based dosage regimen based on developmental pharmacokinetics-pharmacodynamics.

\section{RESULTS}

Study population. A total of 187 patients admitted between 2016 and 2017 were included in this study. All patients fulfilled the inclusion and exclusion criteria, and informed consent was obtained from parents. The mean (standard deviation [SD]) values of weight and postmenstrual age (PMA) of the 187 patients were 2,988.2 (733.3) (range 1,060.0 to 4,580.0) grams and 38.4 (2.6) (range 28.4 to 46.3) weeks, respectively. Patient characteristics are summarized and presented in Table 1.

Model building. A total of 224 amoxicillin concentrations were obtained. The concentrations of the amoxicillin samples ranged from below the limit of quantification $(<\mathrm{LOQ})$ to $73.6 \mu \mathrm{g} / \mathrm{ml}$. Eighteen concentrations were below the LOQ, and these values were censored and replaced with a constant value of $0.25 \mu \mathrm{g} / \mathrm{ml}$, which is equal to half of the LOQ $(0.25 \mu \mathrm{g} / \mathrm{ml})$ in statistical analyses. The profile of concentration versus time is presented in Fig. 1.

In this study, a two-compartment model with first-order elimination was determined as the best fit for the data. Compared with the one-compartment model (objective 


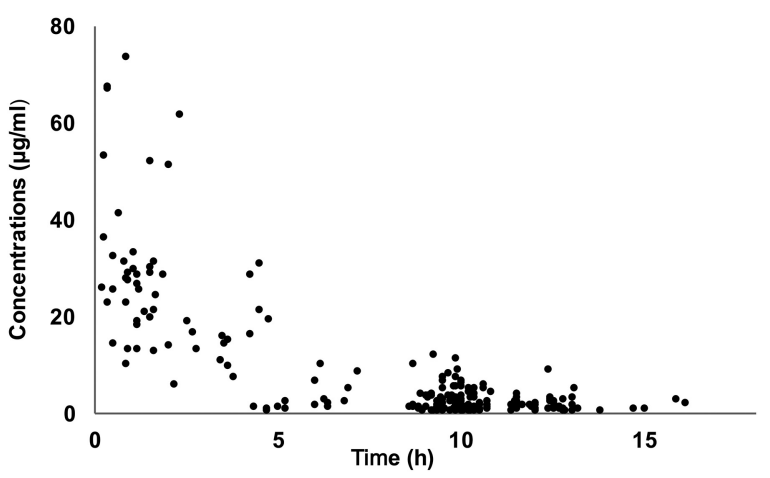

FIG 1 Amoxicillin concentration versus time.

function value [OFV], 871.20 units), the two-compartment (OFV, 754.22 units) model has a low OFV value and residual variability. The model was parameterized in terms of clearance $(C L)$, intercompartment clearance $(Q)$, central volume of distribution $\left(V_{1}\right)$, and peripheral volume of distribution $\left(V_{2}\right)$ of amoxicillin. Residual variability was best described by an exponential model. An exponential model was also used to describe interindividual variability and estimated $V_{2}$ and $\mathrm{CL}$.

Covariate analysis. The current weight $(\mathrm{CW})$ was a priori incorporated into the basic model by the allometric size approach (allometric coefficients were 1 for $V_{1}$ and $V_{2}$ and 0.75 for $C L$ and $Q$ ). As shown in the results, there is a significant drop in OFV ( $\triangle \mathrm{OFV}, 42.5$ units). PMA was the most important covariate of $\mathrm{CL}$ (for postnatal age [PNA], $\triangle$ OFV of 30.1 units; for gestational age [GA], $\triangle$ OFV of 38.6 units) and caused a significant drop ( $\triangle \mathrm{OFV}, 49.5$ units). The combination of GA and PNA proved to be superior to use of PMA alone ( $\triangle \mathrm{OFV}, 83.6$ units). Other covariates were implemented but did not result in a further decrease in the OFV.

The parameter estimates of the final pharmacokinetic model are summarized and presented in Table 2. The weight-normalized volume of distribution (sum of $V_{1}$ and $V_{2}$ )

TABLE 2 Population pharmacokinetic parameters of amoxicillin and bootstrap results

\begin{tabular}{|c|c|c|c|c|}
\hline \multirow[b]{2}{*}{ Parameter $^{a}$} & \multicolumn{2}{|l|}{ Full data set } & \multicolumn{2}{|c|}{ Bootstrap analysis } \\
\hline & Final estimate & RSE $(\%)^{b}$ & Sample median & $\begin{array}{l}\text { Confidence interval } \\
\text { (5th to } 95 \text { th percentile) }\end{array}$ \\
\hline \multicolumn{5}{|c|}{$V_{1}$ (liters) $=\theta 1 \times(\mathrm{CW} / 3,210)$} \\
\hline \multicolumn{5}{|c|}{$V_{2}$ (liters) $=\theta 2 \times(\mathrm{CW} / 3,210)$} \\
\hline Value for $\theta 2$ & 2.42 & 28.30 & 2.43 & $1.40-4.49$ \\
\hline \multicolumn{5}{|c|}{$Q($ liters $/ \mathrm{h})=\theta 3 \times(\mathrm{CW} / 3210)^{0.75}$} \\
\hline Value for $\theta 3$ & 0.17 & 23.70 & 0.16 & $0.10-0.23$ \\
\hline \multicolumn{5}{|c|}{$\mathrm{CL}($ liters $/ \mathrm{h})=\theta 4 \times(\mathrm{CW} / 3210)^{0.75} \times F_{\text {age }}$} \\
\hline Value for $\theta 4$ & 0.81 & 7.50 & 0.81 & $0.70-0.92$ \\
\hline \multicolumn{5}{|c|}{$F_{\text {age }}=(\mathrm{GA} / 38.14)^{\theta 5} \times(\mathrm{PNA} / 7)^{\theta 6}$} \\
\hline $\mathrm{CL}$ & 40.00 & 33.50 & 38.47 & $27.64-48.66$ \\
\hline Residual variability (\%) & 35.00 & 13.60 & 34.93 & $30.21-39.50$ \\
\hline
\end{tabular}

$a V_{1}$, central volume of distribution; $V_{2}$, peripheral volume of distribution; $Q$, intercompartment clearance; $C L$, clearance; $C W$, current weight in grams; $G A$, gestational age in weeks; PNA, postnatal age in days; In our population, 3,210 g, 38.1 weeks, and 7 days are the median current weight (day of the study), gestational age, postnatal age values, respectively.

${ }^{b}$ Relative standard error. 

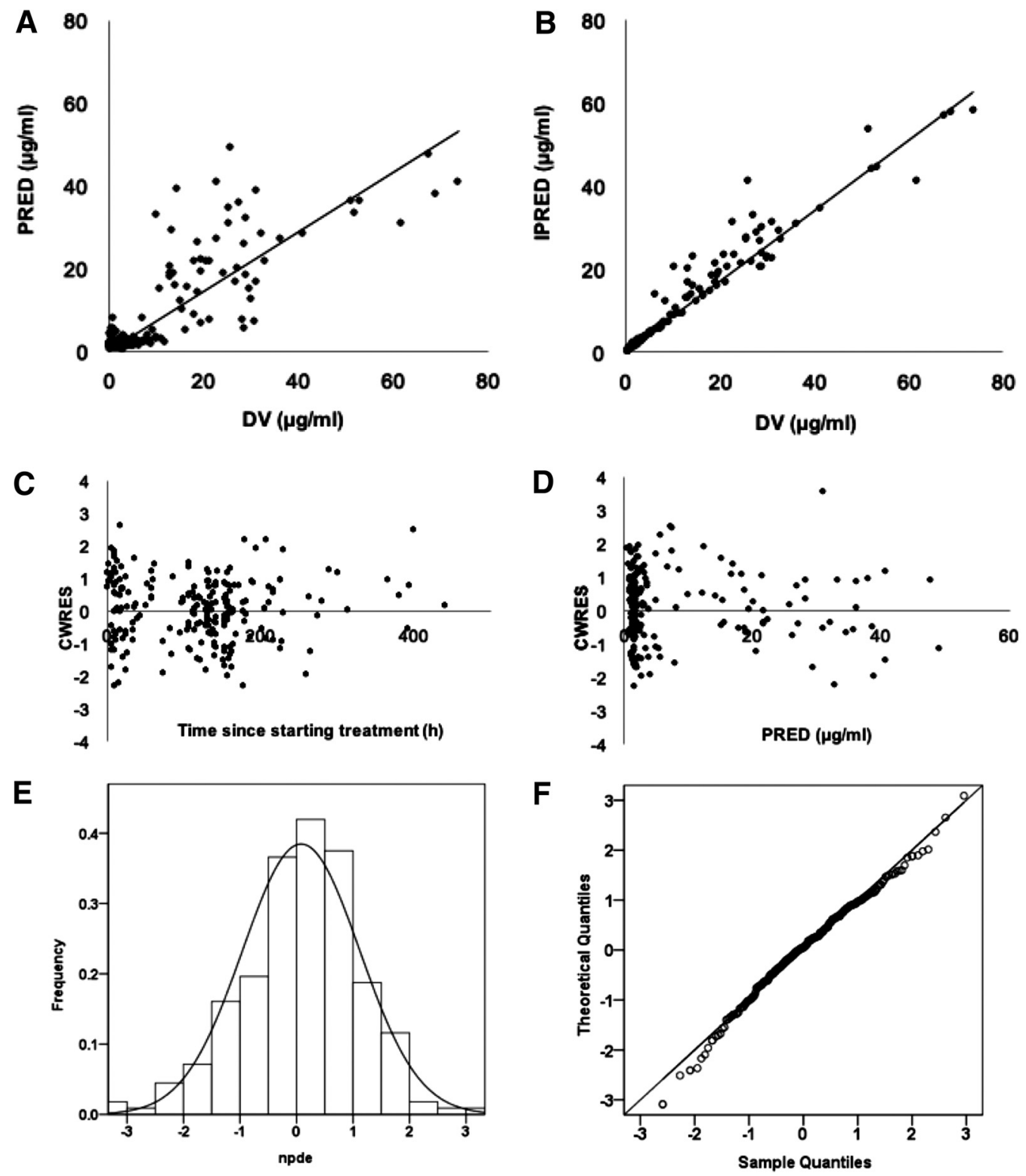

FIG 2 Model evaluation for amoxicillin. (A) PRED versus DV. (B) IPRED versus DV. (C) CWRES versus time. (D) CWRES versus PRED. (E) Histogram of the distribution of the NPDE. (F) QQ plot of the NPDE versus the theoretical $N(0,1)$ distribution.

and CL median values at a steady state are 1.21 (range, 0.69 to 2.05 ) liters $/ \mathrm{kg}$ and 0.25 (range, 0.07 to 0.59 ) liters $/ \mathrm{h} / \mathrm{kg}$, respectively. For the neonates and young infants, the $\mathrm{CL}$ of amoxicillin increased allometrically with current weight.

Internal model validation. Reliable goodness-of-fit results of the final model of amoxicillin are reflected by model diagnostics. As presented in Fig. $2 A$ and $B$, the predictions are unbiased. No trends were observed in the diagnostic plots of conditional weighted residuals (CWRES) versus the population prediction (PRED) and time (Fig. 2C and D).

In addition, the bootstrap analysis presented a stable and reliable result of the final model. The values calculated from the developed model agreed with the median parameter estimated values obtained from the bootstrap procedure. The normalized prediction distribution errors (NPDEs) are shown in Fig. 2E and F, which indicate a good fit of the model to individual data. The distribution and histogram of NPDEs met a criterion of $N(0,1)$ distribution and density. The mean value and variance of the NPDE were 0.08 and 1.08 , respectively. 
TABLE 3 Population pharmacokinetic parameters of final model and total model

\begin{tabular}{|c|c|c|c|c|}
\hline \multirow[b]{2}{*}{ Parameter ${ }^{a}$} & \multicolumn{2}{|c|}{ Final model value } & \multicolumn{2}{|c|}{ Total model value ${ }^{c}$} \\
\hline & Final estimate & $\overline{\text { RSE }(\%)^{b}}$ & Final estimate & RSE (\%) \\
\hline \multicolumn{5}{|c|}{$V_{1}$ (liters) $=\theta 1 \times(\mathrm{CW} / 3210)$} \\
\hline Value for $\theta 1$ & 1.48 & 7.80 & 1.64 & 7.60 \\
\hline \multicolumn{5}{|c|}{$V_{2}$ (liters) $=\theta 2 \times(C W / 3210)$} \\
\hline Value for $\theta 2$ & 2.42 & 28.30 & 2.18 & 26.60 \\
\hline \multicolumn{5}{|c|}{$Q($ liters $/ \mathrm{h})=\theta 3 \times(\mathrm{CW} / 3210)^{0.75}$} \\
\hline Value for $\theta 3$ & 0.17 & 23.70 & 0.17 & 20.10 \\
\hline \multicolumn{5}{|c|}{$\mathrm{CL}($ liters $/ \mathrm{h})=\theta 4 \times(\mathrm{CW} / 3210)^{0.75} \times F_{\text {age }}$} \\
\hline Value for $\theta 4$ & 0.81 & 7.50 & 0.80 & 5.60 \\
\hline \multicolumn{5}{|c|}{$F_{\text {age }}=(\mathrm{GA} / 38.14)^{\theta 5} \times(\mathrm{PNA} / 7)^{\theta 6}$} \\
\hline Value for $\theta 5$ & 4.19 & 12.60 & 4.07 & 11.10 \\
\hline Value for $\theta 6$ & 0.28 & 20.30 & 0.24 & 21.30 \\
\hline \multicolumn{5}{|c|}{ Interindividual variability (\%) } \\
\hline$V_{2}$ & 80.00 & 55.00 & 86.49 & 42.40 \\
\hline $\mathrm{CL}$ & 40.00 & 33.50 & 45.93 & 23.60 \\
\hline Residual variability (\%) & 35.00 & 13.60 & 33.16 & 12.70 \\
\hline
\end{tabular}

External model validation. The validation sets were collected from two hospitals; one set was collected from Beijing Children's Hospital in 2018. These samples were the opportunistic PK samples. A total of 28 patients were included. The mean PMA and weight of the patients at the time of study were 37.9 (range, 29.3 to 43.4 ; SD, 4.1) weeks and 2,691.8 (range, 1,180.0 to 4,200.0; SD, 824.1) grams, respectively. The other validation set was collected from Beijing Obstetrics and Gynecology Hospital between 2016 and 2017. These samples were the therapeutic drug monitoring (TDM) samples. A total of 20 patients were included in the data set. The mean PMA and weight of the 20 patients at the time of study were 38.6 (range, 35.0 to 42.3 ; SD, 1.7) weeks and 3,163.3 (range, 1,940.0 to 4,050.0; SD, 623.7) grams, respectively. The mean values of the mean prediction error (MPE) percent and of the mean absolute prediction error (MAE) percent values are 4.0 (SD, 22.4) and 13.8 (SD, 18.0), respectively, while the percentage of the number of patients with MPE and MAE within the range of \pm 20 and $\pm 30 \%$ were $80 \%$ and $93 \%$, respectively.

All of the data (original data set and validation data set) were used to build a new model. Compared with the original final model, the final estimates of the new model showed a slight difference (results are presented in Table 3).

Dosing regimen evaluation and optimization. During $70 \%$ of the dosage interval, the drug concentration of more than $70 \%$ of patients was above the MIC target. The target attainment rates as a function of the simulated dose for standard MIC susceptibility breakpoints are shown in Fig. 3.

For early-onset sepsis (EOS) (PNA of $<3$ days), the currently used dosage regimen ( $25 \mathrm{mg} / \mathrm{kg}$ BID) resulted in $99.0 \%$ of premature neonates (GA of $<37$ weeks) and $87.3 \%$ of term neonates (GA of $\geq 37$ weeks) achieving the pharmacodynamic target, using the MIC breakpoint of $1 \mathrm{mg} /$ liter. For late-onset sepsis (LOS) (PNA of $\geq 3$ days), $86.1 \%$ of premature neonates treated with $25 \mathrm{mg} / \mathrm{kg}$ three times daily (TID) and $79.0 \%$ of term neonates receiving $25 \mathrm{mg} / \mathrm{kg}$ four times daily (QID) reached the pharmacodynamic target of the MIC susceptibility breakpoint of $2 \mathrm{mg} /$ liter in accordance with the standard EUCAST value. 


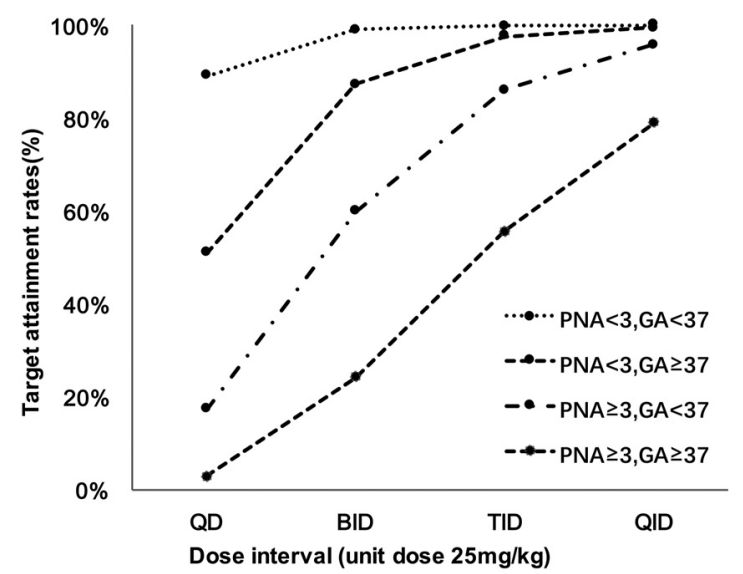

FIG 3 Dosing regimen optimization. For EOS (PNA of $<3$ days), the pharmacodynamic target of different dosage regimens was above the MIC breakpoint of $1 \mathrm{mg} /$ liter. For LOS (PNA of $\geq 3$ ), the MIC breakpoint was $2 \mathrm{mg} / \mathrm{liter}$.

\section{DISCUSSION}

In this study, we reported on the amoxicillin population pharmacokinetics of neonates through the use of a large sample size $(n=187)$ that covered the entire age range of neonates and young infants (PMA of 28.4 to 46.3 weeks). Our results show that the best-fit model to describe the pharmacokinetic data was a two-compartment model with first-order elimination based on current weight, postnatal age, and gestational age.

In our study, the estimated mean CL value is 0.11 liters $/ \mathrm{h} / \mathrm{kg}$ for premature neonates. This is in agreement with previously published values $(0.053$ to 0.18 liters $/ \mathrm{h} / \mathrm{kg})(2,5-7)$. For term neonates, the estimated mean CL value in our study is 0.27 liters $/ \mathrm{h} / \mathrm{kg}$. Since amoxicillin is principally eliminated through a renal route, renal anatomical and functional maturation is considered to have a probable primary influence on amoxicillin clearance and dosing in newborns. When the factor of current weight is taken into consideration through an allometric scaling method, the independent impact of GA and PNA on clearance shows that antenatal and postnatal renal maturation has a crucial influence on amoxicillin clearance.

Developmental pharmacokinetics-pharmacodynamics, microbiology, and safety should be taken into consideration in order to optimize the dosing of antimicrobial therapies (9). The $f T_{>\text {MIC }}$ is an important pharmacokinetic-pharmacodynamic parameter that is correlated with the bacteriological and clinical efficacy of $\beta$-lactam antibiotics $(10,11)$. For adults, it is generally accepted that the percentage of the dosing interval that the drug concentrations is above the MIC ( $T_{\text {MIC }}$ ) is at least $40 \%$ to $50 \%$ (12). Neonates should be regarded as being in an immunocompromised state $(13,14)$ and need a higher $T_{\text {MIC }}$ target in order to avoid the induction of antibiotic resistance and to ensure efficacy $(15,16)$. In our study, a $T_{\text {MIC }}$ target of $70 \%$ of the dosing interval was selected as a more conservative endpoint.

Considering microbiological aspects, the optimum dose of amoxicillin is expected to cover the most common pathogenic bacteria that cause neonatal sepsis. Neonatal sepsis can be categorized as EOS and LOS. The pathogens for each kind of sepsis, as well as the pharmacokinetics, are different. Streptococcus agalactiae and Listeria monocytogenes are the most important neonatal pathogens causing EOS (17). According to EUCAST guidelines, the MIC range of amoxicillin for Streptococcus agalactiae and Listeria monocytogenes is $0.25 \mathrm{mg} /$ liter to $1.0 \mathrm{mg} /$ liter, respectively. Thus, a target MIC of $1 \mathrm{mg} /$ liter was selected as a breakpoint for the determination of the $T_{\text {MIC }}$ for the treatment of EOS. For LOS, various pathogens could cause disease. Therefore, a non-species-related MIC of 2 mg/liter was chosen as the breakpoint for dosing optimization. 
Useful models are expected to precisely describe the original-build data set. Moreover, in order to establish an evidence-based dosage regimen for the safe and effective administration in neonates, pharmacokinetic-pharmacodynamic models are required to predict the expected concentrations, effects, and variability in patients with similar biological and clinical characteristics. Thus, external evaluation is an essential supplementary procedure (18). The predictive performance of the model we developed was confirmed using external validation, making it reliable in dosing prediction.

However, our study has some limitations. Serum creatinine concentration, an indicator of renal function, did not display a significant influence on amoxicillin clearance in our research. This can be explained by the narrow range of creatinine values. Additionally, since residual maternally derived creatinine could have an impact on its concentration, creatinine is not the best predictor of renal function in neonates $(18,19)$. As a newer endogenous renal biomarker, cystatin $C$ (CysC) has been previously shown to be a good renal biomarker in children with sepsis (20). Thus, the potential impact of CysC in clearance will need to be investigated in further studies. In addition, as we do not have neonates undergoing hypothermia, the impact of hypothermia on amoxicillin pharmacokinetics and dose cannot be evaluated as a covariant in our present analysis.

During the study stage, all neonates who fulfilled inclusion and exclusion criteria were included in the study. However, we did not find extreme preterm cases. This is a limitation of this study.

Conclusion. The population pharmacokinetics of amoxicillin was evaluated through a large sample size that covered the entire age range of neonates and young infants. Current weight, GA, and PNA had a significant impact on amoxicillin pharmacokinetics. Evidence-based dosing regimens, based on age and pathogens, were established based on developmental pharmacokinetics-pharmacodynamics.

\section{MATERIALS AND METHODS}

Study design. This study is a prospective, multicenter, open-label pharmacokinetic study of amoxicillin, conducted at Beijing Obstetrics and Gynecology Hospital, Beijing Children's Hospital, and Shandong Provincial Qianfoshan Hospital. Inclusion criteria included neonatal intensive care unit patients with a postmenstrual age of $<48$ weeks, use of amoxicillin as part of regular antimicrobial treatment, and written parental consent to participate in the study. Patients were excluded based on the following criteria: having received other systemic trial drug therapies, having an expected survival time estimated to be less than the length of the treatment cycle, having other factors that the researcher considered unsuitable for inclusion. The ethics board of each institution approved this study.

Amoxicillin, $25 \mathrm{mg} / \mathrm{kg}$ twice daily (BID), was administered as an intravenous bolus for over $5 \mathrm{~min}$ or as an infusion for over $30 \mathrm{~min}$. An opportunistic sampling design was chosen for the collection of pharmacokinetic samples (21). The times of sampling and infusion were recorded accurately. Only samples that had validated sampling information were included. In the clinical practice, blood samples were collected and centrifuged at $1,500 \times g$ for $10 \mathrm{~min}$ at $4^{\circ} \mathrm{C}$. Plasma samples were stored at $-80^{\circ} \mathrm{C}$

Analytical method for amoxicillin. Amoxicillin concentrations were determined through separation analyses using high-performance liquid chromatography (HPLC), with tinidazole as an internal standard. The calibration curve for amoxicillin was over a concentration range of 0.5 to $20 \mu \mathrm{g} / \mathrm{ml}$. The lower limit of quantification (LLOQ) was $0.5 \mu \mathrm{g} / \mathrm{ml}$. The interday and intraday coefficients of variation (CVs) of quality control samples were $4.30 \%$ and $3.05 \%$, respectively. In this study, free concentration of amoxicillin was not measured. Since the amount of protein binding of amoxicillin in neonates is low (about 10\%) (22), it can be considered negligible in determining our target concentration.

Population pharmacokinetic modeling of amoxicillin. NONMEM, version 7.2 (Icon Development Solutions, USA), the nonlinear mixed effects modeling program, was used to perform pharmacokinetic analysis. In order to evaluate pharmacokinetic (PK) parameters and their variability, the first-order conditional estimation method with interaction was applied.

An exponential model for interindividual variability was also applied in this study. The exponential model can be expressed as follows:

$$
\theta_{i}=\theta_{\text {mean }}{ }^{*} \exp (\eta \mathrm{i})
$$

where $\theta_{\text {mean }}$ is the typical value for the population (a fixed effect), $\eta$ i is the symmetrically distributed variability between subjects, following the normal distribution (variance $\omega 2$ and mean zero), and $\theta_{i}$ is the individual pharmacokinetic parameter value for the ith subject.

The forward-selection process was applied to the covariate analysis. In a forward-selection process and a backward-elimination process, covariates are considered in various rounds, with a single covariate added at the conclusion of each step. 
The impact of birth weight (BW), gestational age (GA), postmenstrual age (PMA), current weight (CW), postnatal age (PNA), and serum creatinine concentration (pharmacokinetic samples were collected within $48 \mathrm{~h}$ ) were investigated and added to the model.

If the objective function value (OFV) decrease was $>3.84$ compared with value of the basic model, the covariate was considered to have a significant impact $(P<0.05)$. The covariates that had a significant impact were added together into the model. In the next step, covariates were successively removed from the model. In the backward-elimination process, the covariate was finally retained when the increase in OFV was above 6.635

Model validation. Statistical and graphical criteria were used to validate the performance of the model. Both internal validation and external validation were performed to verify the predictive performance of the model.

(i) Internal model validation. Scatterplots of observed (dependent variable [DV]) versus population prediction (PRED), DV versus individual prediction (IPRED), conditional weighted residuals (CWRES) versus PRED, and CWRES versus time were used to validate the model (28).

Nonparametric bootstrapping with replacement and resampling was performed to evaluate the performance and stability of the final model. The values of estimated parameters of the original data set were compared with parameters obtained from the bootstrap procedure, in which resampling was repeated 1,000 times. PsN (version 2.30) was used to execute the entire procedure in an automated fashion (23). Normalized prediction distribution error (NPDE) analysis was performed to further assess the final model. A total of 1,000 data sets were simulated using final population model parameters. A quantile-quantile (QQ) plot and histogram of the NPDE were provided by the open-source statistical package $R$ (24). The NPDE distribution was expected to follow a normal $[N(0,1)]$ distribution.

(ii) External model validation. The independent data set obtained from routine therapeutic drug monitoring was used to validate the model. The validation sets were collected from two hospitals; one set was from Beijing Children's Hospital of patients admitted in 2018, and these samples were the opportunistic PK samples. The other data set was obtained from Beijing Obstetrics and Gynecology Hospital of patients admitted between 2016 and 2017, and these samples were the TDM samples. This study was approved by the IRB of Beijing Obstetrics and Gynecology Hospital, Beijing Children's Hospital, and Shandong Provincial Qianfoshan Hospital for the use of these data. There was sufficient information on patients of the validation data sets. This information included basic physiological information, dosing information, sampling information, and covariate information.

Mean absolute prediction (MAE) and mean prediction error (MPE) were applied to calculate bias and imprecision of predictive performance (25) (equations 2 and 3). In addition, the number of patients with MAE and MPE values within the range of \pm 20 and $\pm 30 \%$ were calculated (26)

$$
\begin{gathered}
\mathrm{MAE} \%=\frac{1}{N} \sum\left|\frac{\mathrm{PRED}_{i}-\mathrm{OBS}_{i}}{\mathrm{OBS}_{i}}\right| \times 100 \% \\
\mathrm{MPE} \%=\frac{1}{N} \sum \frac{\mathrm{PRED}_{i}-\mathrm{OBS}_{i}}{\mathrm{OBS}_{i}} \times 100 \%
\end{gathered}
$$

Dosing regimen evaluation and optimization. Amoxicillin has a time-dependent effect on bacteria, for which the pharmacokinetics-pharmacodynamics relationship is the time period in which the free antimicrobial drug concentration is above MIC $\left(f T_{>M I C}\right)$. According to the European Committee on Antimicrobial Susceptibility Testing (EUCAST), common pathogens that cause early-onset sepsis (EOS) and late-onset sepsis (LOS), along with relevant MIC values were also considered for dose optimization (27). For EOS, the MIC range of amoxicillin for the most common pathogens, Streptococcus agalactiae and Listeria monocytogenes, was between 0.25 and $1.0 \mathrm{mg} /$ /iter. For LOS, because various different pathogens cause LOS, a non-species-related MIC of $2 \mathrm{mg} /$ liter was selected as the PK-PD breakpoint.

The neonatal dosage simulation of amoxicillin was on a milligram/kilogram basis, according to age group. Monte Carlo simulations were performed using parameter estimates obtained from the final model. The original data set was simulated 100 times, and the time above the MIC was calculated for each original patient. The original patients were used in the simulation in order to make sure that the population distribution characteristics were the same. In the event the current dosing regimen was found to be underdosed, the virtual patient was administered the optimal dosing regimen with increased frequency and/or dose (29-31). Therefore, different dosing regimens $(25,30,35,40,45$, and $50 \mathrm{mg} / \mathrm{kg}$ twice daily $[B I D]$ to four times daily [QID]) were simulated. For each dosing regimen, the probability of target attainment was calculated to optimize antimicrobial therapy.

\section{ACKNOWLEDGMENTS}

We thank all the children and their families for participating in this study.

This study was supported by National Science and Technology Major Projects for Major New Drugs Innovation and Development (2017ZX09304029-002), the Young Taishan Scholars Program of Shandong Province and Qilu Outstanding Program of Shandong University, Capital Health Research and Development of Special Grant (2016-1-2092) and China Postdoctoral Science Foundation (2015M582102), and Capital's Funds for Health Improvement and Research (2018-4-1142).

We declare that we have conflicts of interest relevant to this article. 


\section{REFERENCES}

1. Sutherland R, Croydon EA, Rolinson GN. 1972. Amoxycillin: a new semisynthetic penicillin. Br Med J 3:13-16. https://doi.org/10.1136/bmj.3 .5817.13.

2. Deboer JJH, Vandenanker JN, Vogel M, Goessens WHF, Schoemaker RC, Degroot R. 1995. Amoxicillin pharmacokinetics in preterm infants with gestational ages of less-than 32 weeks. Antimicrob Agents Chemother 39:431-434. https://doi.org/10.1128/AAC.39.2.431.

3. Hantson P, Leonard F, Maloteaux JM, Mahieu P. 1999. How epileptogenic are the recent antibiotics? Acta Clin Belg 54:80-87. https://doi.org/10 .1080/17843286.1999.11754213.

4. Leroux S, Zhao W, Betremieux P, Pladys P, Saliba E, Jacqz-Aigrain E, French Society of Neonatology. 2015. Therapeutic guidelines for prescribing antibiotics in neonates should be evidence-based: a French national survey. Arch Dis Child 100:394-398. https://doi.org/10.1136/ archdischild-2014-306873.

5. Pullen J, Stolk LML, Nieman FHM, Degraeuwe PLJ, van Tiel FH, Zimmermann LJI. 2006. Population pharmacokinetics and dosing of amoxicillin in (pre)term neonates. Ther Drug Monit 28:226-231. https://doi.org/10 .1097/01.ftd.0000198648.39751.11.

6. Charles BG, Preechagoon Y, Lee TC, Steer PA, Flenady VJ, Debuse N. 1997. Population pharmacokinetics of intravenous amoxicillin in very low birth weight infants. J Pharm Sci 86:1288-1292. https://doi.org/10 .1021 /js970068l.

7. Pullen J, Driessen M, Stolk LM, Degraeuwe PL, van Tiel FH, Neef C, Zimmermann LJ. 2007. Amoxicillin pharmacokinetics in (preterm) infants aged 10 to 52 days: effect of postnatal age. Ther Drug Monit 29:376-380. https://doi.org/10.1097/FTD.0b013e318067de5c.

8. Bijleveld YA, Mathot RAA, van der Lee JH, Groenendaal F, Dijk PH, van Heijst A, Simons SHP, Dijkman KP, van Straaten HLM, Rijken M, Zonnenberg IA, Cools F, Zecic A, Nuytemans DHGM, van Kaam AH, de Haan TR, PharmaCool Study Group. 2018. Population pharmacokinetics of amoxicillin in term neonates undergoing moderate hypothermia. Clin Pharmacol Ther 103:458-467. https://doi.org/10.1002/cpt.748.

9. Barker Cl, Germovsek E, Hoare RL, Lestner JM, Lewis J, Standing JF. 2014. Pharmacokinetic/pharmacodynamic modelling approaches in paediatric infectious diseases and immunology. Adv Drug Deliv Rev 73:127-139. https://doi.org/10.1016/j.addr.2014.01.002.

10. Jacobs MR. 2001. Optimisation of antimicrobial therapy using pharmacokinetic and pharmacodynamic parameters. Clin Microbiol Infect 7:589-596. https://doi.org/10.1046/j.1198-743x.2001.00295.x.

11. Levison ME, Levison JH. 2009. Pharmacokinetics and pharmacodynamics of antibacterial agents. Infect Dis Clin North Am 23:791-815. https://doi .org/10.1016/j.idc.2009.06.008.

12. Craig WA. 2001. Does the dose matter? Clin Infect Dis 33:S233-S237. https://doi.org/10.1086/321854.

13. Wynn JL, Levy O. 2010. Role of innate host defenses in susceptibility to early-onset neonatal sepsis. Clin Perinatol 37:307-337. https://doi.org/ 10.1016/j.clp.2010.04.001.

14. Wynn J, Cornell TT, Wong HR, Shanley TP, Wheeler DS. 2010. The host response to sepsis and developmental impact. Pediatrics 125: 1031-1041. https://doi.org/10.1542/peds.2009-3301.

15. MacGowan AP. 2004. Elements of design: the knowledge on which we build. Clin Microbiol Infect 10:6-11. https://doi.org/10.1111/j.1470-9465 .2004.00863.x

16. Craig WA. 2003. Basic pharmacodynamics of antibacterials with clinical applications to the use of beta-lactams, glycopeptides, and linezolid. Infect Dis Clin North Am 17:479. https://doi.org/10.1016/S0891-5520(03)00065-5.

17. USCAST. 2016. Breakpoint tables for interpretation of MICs and zone diameters (v2.1). U.S. Committee on Antimicrobial Susceptibility Testing
Silverton, OR. https://app.box.com/s/2klcl72f3zg1swe1jr6bopu67 q6s39ha.

18. Zhao W, Kaguelidou F, Biran V, Zhang D, Allegaert K, Capparelli EV Holford N, Kimura T, Lo YL, Peris JE, Thomson A, van den Anker JN, Fakhoury M, Jacqz-Aigrain E. 2013. External evaluation of population pharmacokinetic models of vancomycin in neonates: the transferability of published models to different clinical settings. Br J Clin Pharmacol 75:1068-1080. https://doi.org/10.1111/j.1365-2125.2012.04406.x.

19. Abitbol CL, DeFreitas MJ, Strauss J. 2016. Assessment of kidney function in preterm infants: lifelong implications. Pediatr Nephrol 31:2213-2222. https://doi.org/10.1007/s00467-016-3320-x.

20. Di Nardo M, Ficarella A, Ricci Z, Luciano R, Stoppa F, Picardo S, Picca S, Muraca M, Cogo P. 2013. Impact of severe sepsis on serum and urinary biomarkers of acute kidney injury in critically ill children: an observational study. Blood Purif 35:172-176. https://doi.org/10.1159/000346629.

21. Leroux $S$, Turner MA, Guellec $C B$, Hill $H$, van den Anker JN, Kearns GL, Jacqz-Aigrain E, Zhao W, TINN (Treat Infections in NeoNates) and GRiP (Global Research in Paediatrics) Consortiums. 2015. Pharmacokinetic studies in neonates: the utility of an opportunistic sampling design. Clin Pharmacokinet 54:1273-1285. https://doi.org/10.1007/s40262-015-0291-1.

22. Pullen J, Stolk LML, Nieman FHM, Degraeuwe PLJ, van Tiel FH, Zimmermann LJI. 2006. Population pharmacokinetics and dosing of amoxicillin in (pre)term neonates-response to van den Anker. Ther Drug Monit 28:816-817. https://doi.org/10.1097/01.ftd.0000255010.68239.63.

23. Lindbom L, Ribbing J, Jonsson EN. 2004. Perl-speaks-NONMEM (PsN)-a Perl module for NONMEM related programming. Comput Methods Programs Biomed 75:85-94. https://doi.org/10.1016/j.cmpb.2003.11.003.

24. Comets E, Brendel K, Mentre F. 2008. Computing normalised prediction distribution errors to evaluate nonlinear mixed-effect models: the npde add-on package for R. Comput Methods Programs Biomed 90:154-166. https://doi.org/10.1016/j.cmpb.2007.12.002.

25. Ding JJ, Wang Y, Lin WW, Wang CL, Zhao LM, Li XG, Zhao ZG, Miao LY, Jiao Z. 2015. A population pharmacokinetic model of valproic acid in pediatric patients with epilepsy: a non-linear pharmacokinetic model based on protein-binding saturation. Clin Pharmacokinet 54:305-317. https://doi.org/10.1007/s40262-014-0212-8.

26. van der Meer AF, Marcus MAE, Touw DJ, Proost JH, Neef C. 2011. Optimal sampling strategy development methodology using maximum a posteriori bayesian estimation. Therapeutic Drug Monit 33:133-146:1. https:// doi.org/10.1097/FTD.0b013e31820f40f8.

27. EUCAST. 2016. Antimicrobial susceptibility testing. Clinical breakpointsbacteria (v 6.0). http://www.eucast.org/ast_of_bacteria/previous_versions _of_documents/.

28. Hooker AC, Staatz CE, Karlsson MO. 2007. Conditional weighted residuals (CWRES): a model diagnostic for the FOCE method. Pharm Res 24: 2187-2197. https://doi.org/10.1007/s11095-007-9361-x.

29. Drusano GL. 2003. Prevention of resistance: a goal for dose selection for antimicrobial agents. Clin Infect Dis 36:S42-S50. https://doi.org/10.1086/ 344653.

30. Falagas ME, Tansarli GS, Ikawa K, Vardakas KZ. 2013. Clinical outcomes with extended or continuous versus short-term intravenous infusion of carbapenems and piperacillin/tazobactam: a systematic review and meta-analysis. Clin Infect Dis 56:272-282. https://doi.org/10.1093/cid/ cis857.

31. Roberts JA, Abdul-Aziz MH, Davis JS, Dulhunty JM, Cotta MO, Myburgh J, Bellomo R, Lipman J. 2016. Continuous versus intermittent betalactam infusion in severe sepsis. A meta-analysis of individual patient data from randomized trials. Am J Respir Crit Care Med 194:681-691. https://doi.org/10.1164/rccm.201601-0024OC. 